\title{
Phylogenetic Analysis of Fusobacterium prausnitzii Based upon the 16S rRNA Gene Sequence and PCR Confirmation
}

\author{
RONG-FU WANG, WEI-WEN CAO, AND CARL E. CERNIGLIA* \\ Microbiology Division, National Center for Toxicological Research, \\ Food and Drug Administration, Jefferson, Arkansas 72079
}

\begin{abstract}
In order to develop a PCR method to detect Fusobacterium prausnitzii in human feces and to clarify the phylogenetic position of this species, its 16S rRNA gene sequence was determined. The sequence described in this paper is different from the 16S rRNA gene sequence of $F$. prausnitzii in the GenBank database (accession number M58682). A PCR assay based on the new sequence is specific for $F$. prausnitzii, and the results of this assay confirmed that $F$. prausnitzii is the most common species in human feces. However, a PCR assay based on the original GenBank sequence was negative when it was performed with two strains of $F$. prausnitzii obtained from the American Type Culture Collection. A phylogenetic tree based on the new 16S rRNA gene sequence was constructed. On this tree $F$. prausnitzii was not a member of the Fusobacterium group but was closer to some Eubacterium spp. and located between Clostridium "clusters III and IV" (M. D. Collins, P. A. Lawson, A. Willems, J. J. Cordoba, J. Fernandez-Garayzabal, P. Garcia, J. Cai, H. Hippe, and J. A. E. Farrow, Int. J. Syst. Bacteriol. 44:812-826, 1994).
\end{abstract}

Fusobacterium prausnitzii is one of the most common organisms in human gastrointestinal tracts. In previous studies it was found that $F$. prausnitzii accounted for more than $7 \%$ of the human fecal microflora and ranked second in relative frequency among bacterial species isolated from human feces $(6$, 7).

Fusobacterium spp. are anaerobic, gram-negative, straight, curved, or helical rod-shaped organisms which belong to the family Bacteroidaceae (7). Lawson et al. (4) investigated the phylogenetic relationships of 14 members of the genus Fusobacterium, but $F$. prausnitzii was not included in this study. Neefs et al. (8) described a taxonomic classification of all Fusobacterium species based on the results of a comparison of their small-subunit rRNA sequences. These authors found that on their bacterial subtree the Fusobacterium spp. did not belong to any cluster, and therefore these organisms were listed separately in a group designated "Fusobacterium and relatives." All Fusobacterium species except $F$. prausnitzii were listed as members of this group, and $F$. prausnitzii was listed as a member of a group that contained gram-positive organisms and their relatives which have high $\mathrm{G}+\mathrm{C}$ contents (8). Nicholson et al. (9) reported that the results of an analysis of $16 \mathrm{~S}$ rRNA sequences indicated that $F$. prausnitzii is more closely related to the gram-positive organisms Propionibacterium acnes and Actinomyces israelii than to Fusobacterium spp. These authors pointed out that this independent finding is consistent with the findings of Neefs et al.; the 16S rRNA sequence of $F$. prausnitzii used in this study was the sequence deposited in the GenBank database under accession number M58682.

We have been developing molecular methods to monitor intestinal microflora to study the effects of food additives, xenobiotic compounds, and pharmaceutical drugs on the human colonic microflora (10). In a study to develop a PCR method for detection of $F$. prausnitzii in human feces, the $16 \mathrm{~S}$ rRNA gene sequence deposited in the GenBank database (accession number M58682) was used first. We designed a pair of PCR primers on the basis of this sequence. The sequence of forward primer oFPR-1 is GCTTTTGTGGGGGCTGAGT

\footnotetext{
${ }^{*}$ Corresponding author. Phone: (501) 543-7341. Fax: (501) 543-
}

(base positions 82 to 100 ), and the sequence of reverse primer oFPR-2 is CTGATAAGCCGCGAGTCCA (base positions 246 to 228). When we used this primer set, we did not obtain positive PCR results with either $F$. prausnitzii ATCC 27766 or $F$. prausnitzii ATCC 27768 . We wondered if the sequence deposited in the GenBank database might be incorrect; therefore, we partially sequenced the $16 \mathrm{~S}$ rRNA gene of $F$. prausnitzii ATCC 27768 and designed a new set of PCR primers on the basis of the resulting sequence information. The sequence of forward primer FPR-1 was AGATGGCCTCGCGTCCGA (base positions 222 to 239), and the sequence of reverse primer FPR-2 was CCGAAGACCTTCTTCCTCC (base positions 420 to 402). PCR assays in which we used this primer set gave positive results with $F$. prausnitzii ATCC 27766 and ATCC 27768. In addition, after reviewing the results of the studies of Neefs et al. (8) and Nicholson et al. (9), we decided that the entire 16S rRNA gene sequence of $F$. prausnitzii ATCC 27766 should be determined.

Amplification and sequencing of the 16S rRNA gene. A 1.5 -ml pure culture of $F$. prausnitzii was centrifuged to pellet the bacterial cells. The cells were washed twice with phosphate-buffered saline (PBS) and once with autoclaved distilled water and then resuspended in $0.1 \mathrm{ml}$ of distilled water. The cells $(10 \mu \mathrm{l})$ were diluted $1: 10$ with $90 \mu \mathrm{l}$ of $1 \%$ Triton X-100, heated at $100^{\circ} \mathrm{C}$ for $5 \mathrm{~min}$, immediately cooled in ice water, and then subjected to PCR amplification without isolating the DNA. A $3.5-\mu l$ portion of each sample was added to $45 \mu \mathrm{l}$ of a PCR mixture containing $50 \mathrm{mM}$ Tris- $\mathrm{HCl}(\mathrm{pH} 8.5), 20 \mathrm{mM}$ $\mathrm{KCl}, 3 \mathrm{mM} \mathrm{MgCl}, 0.05 \%$ bovine serum albumin (catalog no. A-4378; Sigma Chemical Co., St. Louis, Mo.), $0.25 \mathrm{mM}$ dATP, $0.25 \mathrm{mM}$ dTTP, $0.25 \mathrm{mM}$ dCTP, $0.25 \mathrm{mM} \mathrm{dGTP}$, each primer at a concentration of $0.25 \mu \mathrm{M}$, and $1.8 \mathrm{U}$ of Taq polymerase. The PCR was performed in a Perkin-Elmer model 480 thermal cycler. The following program was used: one cycle consisting of $95^{\circ} \mathrm{C}$ for $3 \mathrm{~min}$, followed by 35 cycles consisting of $95^{\circ} \mathrm{C}$ for $20 \mathrm{~s}, 55^{\circ} \mathrm{C}$ for $20 \mathrm{~s}$, and $74^{\circ} \mathrm{C}$ for $60 \mathrm{~s}$, and finally one cycle consisting of $74^{\circ} \mathrm{C}$ for $3 \mathrm{~min}$ and $25^{\circ} \mathrm{C}$ for $5 \mathrm{~s}$. The PCR products were separated by electrophoresis in a $1 \%$ agarose gel containing ethidium bromide. The DNA band (about $1.5 \mathrm{~kb}$ ) was excised from the agarose gel under a long-wave UV lamp. The Glassmilk method (Geneclean II kit; Bio 101, Inc., La Jolla, Calif.) was used to recover the DNA as recommended by 
the manufacturer. The sequences of the PCR products were determined directly by using a SequiTherm cycle sequencing kit (Epicentre Technologies, Madison, Wis.) with ${ }^{35}$ S-dATP as recommended by the manufacturer.

The amplification primers used were primers Amp-1 and Amp-2, which were located at the $5^{\prime}$ and $3^{\prime}$ ends of 16 S rRNA gene sequence. These two primers were also used as sequencing primers with the following eight other sequencing primers: primers U2, RU2, U3, RU3, U6, RU6, U7, and RU7, which are located in the internal conserved regions of the $16 \mathrm{~S}$ rRNA gene sequence. The sequences of the primers were as follows: primer Amp-1 (base positions 7 to 27 in the Escherichia coli numbering system [1]), GAGAGTTTGATYCTGGCTCAG; primer Amp-2 (base positions 1538 to 1519), AAGGAGGT GATCCARCCGCA; primer U2 (base positions 355 to 338), TGCTGCCTCCCGTAGGAG; primer RU2 (base positions 338 to 355), CTCCTACGGGAGGCAGCA; primer U3 (base positions 535 to 518), GWATTACCGCGGCKGCTG; primer RU3 (base positions 518 to 535), CAGCAGCCGCGGTAA TAC; primer U6 (base positions 1072 to 1054), ACGAGCT GACGACAGCCAT; primer RÚ6 (base positions 1054 to 1072), ATGGCTGTCGTCAGCTCGT; primer U7 (base positions 1405 to 1387), ACGGGCGGTGTGTACAAGG; and primer RU7 (base positions 1391 to 1407), GTACACACCGC CCGTCA.

First, part of the 16S rRNA gene of $F$. prausnitzii ATCC 27768 was sequenced by using primer U3. The entire $16 \mathrm{~S}$ rRNA gene sequence of $F$. prausnitzii ATCC 27766 was then determined on both strands. The partial sequence of $F$. prausnitzii ATCC 27768 which we determined was exactly the same as the partial sequence of $F$. prausnitzii ATCC 27766.

PCR detection. PCR detection was performed with a model 1605 air thermal cycler (Idaho Technology, Idaho Falls, Idaho). The following conditions were used: one cycle consisting of $94^{\circ} \mathrm{C}$ for $15 \mathrm{~s}$, followed by 35 cycles consisting of $94^{\circ} \mathrm{C}$ for 3 $\mathrm{s}, 50^{\circ} \mathrm{C}$ for $10 \mathrm{~s}$, and $74^{\circ} \mathrm{C}$ for $35 \mathrm{~s}$ at transition speed $\mathrm{S}-9$, and finally one cycle consisting of $74^{\circ} \mathrm{C}$ for $2 \mathrm{~min}$ and $45^{\circ} \mathrm{C}$ for $2 \mathrm{~s}$. The results of our PCR detection analysis performed with selected bacterial species are shown in Table 1 . The primer set consisting of primers oFPR-1 and oFPR-2, which were originally designed by using GenBank sequence M58682, gave negative PCR results with both $F$. prausnitzii ATCC 27766 and $F$. prausnitzii ATCC 27768 . However, the primer set consisting of primers FPR-1 and FPR-2, which were designed by using the 16S rRNA sequence of $F$. prausnitzii determined in this study, gave positive PCR results with both $F$. prausnitzii ATCC 27766 and $F$. prausnitzii ATCC 27768 but negative results with all of the other bacterial strains tested (Table 1). As few as four cells of $F$. prausnitzii in pure culture could be detected by this PCR method (data not shown).

The American Type Culture Collection has only two strains of $F$. prausnitzii (ATCC 27766 and ATCC 27768) which could be used as positive controls. However, the PCR method described above was used to detect $F$. prausnitzii in nine human fecal samples and several animal fecal samples. For each fecal sample preparation, $1 \mathrm{~g}$ (wet weight) of feces was mixed with 9 $\mathrm{ml}$ of PBS (0.05 M; pH 7.4) by inverting and vortexing for 5 to $10 \mathrm{~min}$. The mixed samples were then centrifuged at a low speed $(200 \times g)$ for $5 \mathrm{~min}$ to remove the debris, and this procedure was repeated three times. The upper phase was then centrifuged at $9,000 \times g$ for 3 min to collect the bacterial cells. The cells were washed four times in PBS and once in water, resuspended in $0.1 \mathrm{ml}$ of distilled water, and serially diluted in $100 \mu \mathrm{l}$ of $1 \%$ Triton $\mathrm{X}-100$ for a semiquantitative detection analysis. The samples were heated at $100^{\circ} \mathrm{C}$ for $5 \mathrm{~min}$ and
TABLE 1. PCR results for bacterial strains obtained by using primers based on the new and original sequences

\begin{tabular}{|c|c|c|}
\hline \multirow[b]{2}{*}{ Strain } & \multicolumn{2}{|c|}{ PCR results obtained with ${ }^{a}$ : } \\
\hline & $\begin{array}{l}\text { Primers } \\
\text { FPR-1 and } \\
\text { FPR-2 }{ }^{b}\end{array}$ & $\begin{array}{l}\text { Primers } \\
\text { oFPR-1 and } \\
\text { oFPR-2 }\end{array}$ \\
\hline Fusobacterium prausnitzii ATCC 27768 & + & - \\
\hline Fusobacterium prausnitzii ATCC 27766 & + & - \\
\hline Fusobacterium nucleatum ATCC 25586 & - & - \\
\hline Fusobacterium mortiferum ATCC 9817 & - & - \\
\hline Peptostreptococcus productus AТCC 27340 & $?$ & - \\
\hline Bifidobacterium adolescentis ATCC 15703 & - & ND \\
\hline Bifidobacterium longum ATCC 15707 & - & ND \\
\hline Eubacterium lentum ATCC 25559 & - & - \\
\hline Eubacterium hadrum ATCC 29173 & - & - \\
\hline Bacteroides distasonis ATCC 8503 & - & ND \\
\hline Bacteroides thetaiotaomicron ATCC 29148 & - & ND \\
\hline Bacteroides vulgatus ATCC 8482 & - & - \\
\hline Clostridium clostridiiforme ATCC 25537 & - & - \\
\hline Clostridium perfringens ATCC 13124 & - & ND \\
\hline Salmonella typhimurium ATCC 14028 & - & - \\
\hline Shigella flexneri ATCC 12022 & - & - \\
\hline Yersinia enterocolitica ATCC 27729 & - & ND \\
\hline Lactobacillus acidophilus ATCC 332 & - & ND \\
\hline Propionibacterium acnes ATCC 6919 & - & ND \\
\hline Escherichia coli ATCC 25922 & - & - \\
\hline Actinomyces naeslundii ATCC 12104 & - & ND \\
\hline Klebsiella pneumoniae ATCC 13883 & - & - \\
\hline Pseudomonas aeruginosa ATCC 27853 & - & ND \\
\hline Citrobacter freundii ATCC 8090 & - & ND \\
\hline Enterococcus faecalis ATCC 19433 & - & - \\
\hline Corynebacterium kutscheri ATCC 15677 & - & ND \\
\hline Bacillus subtilis ATCC 6051 & - & ND \\
\hline Streptococcus equi ATCC 9528 & - & ND \\
\hline Proteus vulgaris ATCC 13315 & - & ND \\
\hline Listeria monocytogenes ATCC 35152 & - & ND \\
\hline Vibrio parahaemolyticus ATCC 17802 & - & ND \\
\hline Pasteurella pneumotropica ATCC 35149 & - & ND \\
\hline
\end{tabular}

$a$ The PCR product was 199 bp long. Approximately $10^{5}$ cells of each strain were used for the PCR assay. +, positive; -, negative; ?, a nonspecific reaction occurred, but the size of the PCR product was different from the size of the $F$. prausnitzii product; ND, not determined.

${ }^{b}$ Primers based on the new $16 \mathrm{~S}$ rRNA sequence (the sequence determined in this study).

${ }^{c}$ Primers based on the original 16S rRNA sequence (the sequence whose GenBank accession number is M58682)

immediately cooled in ice water, and $2 \mu l$ of each sample was directly subjected to the PCR assay without isolating the DNA.

Figure 1 shows the results of a PCR analysis to detect $F$. prausnitzii in a fecal sample, in which PCR-amplified DNA bands were obtained from $10^{-1}$ to $10^{-6}$ dilutions of the sample. We also detected 11 other common human intestinal bacterial species in fecal samples by using 11 other specific PCR methods. Our results confirmed that $F$. prausnitzii is one of the most common species in human feces and that the number of cells of this bacterium in feces is 100 to 100,000 times greater than the number of $E$. coli cells (data not shown).

Phylogenetic analysis. The sequence which we determined was sent to the Ribosomal Database Project (RDP) at the University of Illinois for phylogenetic analysis (5). An unrooted tree that included all Fusobacterium species for which $16 \mathrm{~S}$ rRNA gene sequences were available in the RDP database and other closely related bacterial species was produced by the RDP (data not shown). A rooted tree which included some representatives of related taxa was also produced by using the program Lasergene (DANSTAR, Inc., Madison, Wis.) (Fig. 2). 


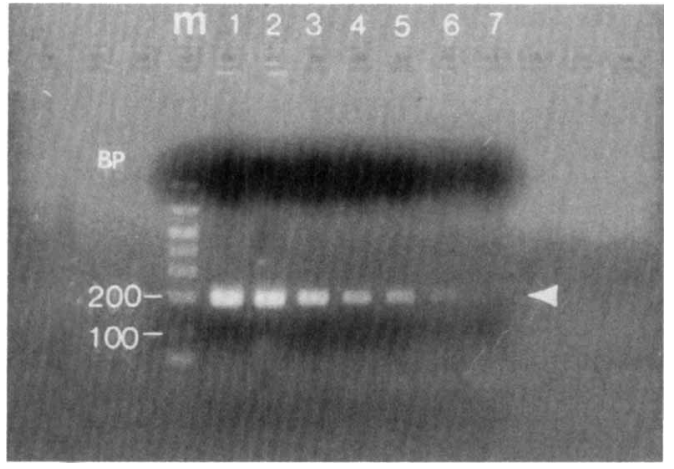

FIG. 1. PCR detection of $F$, prausnitzii in a human fecal sample. The PCR products (199 bp) were separated in a $2 \%$ agarose gel. Lane $\mathrm{m}$, molecular size marker; lanes 1 through 7 , PCR performed with $10^{-1}, 10^{-2}, 10^{-3}, 10^{-4}, 10^{-5}$ $10^{-6}$, and $10^{-7}$ dilutions, respectively.

On both phylogenetic trees, $F$. prausnitzii was not a member of the Fusobacterium group but was closer to some Eubacterium spp. and was located between Clostridium "clusters III and IV" (2). The levels of similarity between the $16 \mathrm{~S}$ rRNA sequence of $F$. prausnitzii determined in this study and the sequences of other Fusobacterium spp. were only $76 \pm 4 \%$. However, the levels of sequence similarity with Eubacterium siraeum, Eubacterium desmolans, and Clostridium cellulosi were 86, 84, and $84 \%$, respectively. These similarity values are not as high as the values obtained for Listeria species (3) and Mycobacterium species (11). The $16 \mathrm{~S}$ rRNA sequences of members of the genera Eubacterium and Clostridium are not as similar as the sequences of members of many better-defined genera, and the taxonomy of these groups is being revised (2). These findings

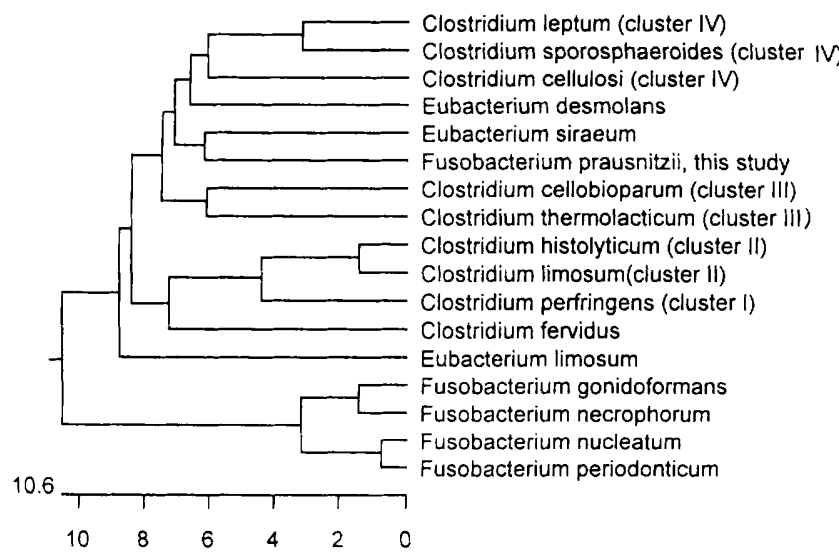

FIG. 2. Phylogenetic tree for $F$. prausnitzii and related species. The tree was constructed by using the Clustal method (DANSTAR program). Clusters I through IV were the clusters described by Collins et al. (2). suggested that $F$. prausnitzii is not affiliated with the genus Fusobacterium. However, formal reclassification of $F$. prausnit$z i i$ is not proposed at this time since there is uncertainty concerning the classification of Clostridium spp. and Eubacterium spp. (2).

Nucleotide sequence accession number. The $16 \mathrm{~S}$ rRNA gene sequence of $F$. prausnitzii ATCC 27766 has been deposited in the EMBL database under accession number X85022.

We thank the RDP for helping to produce a phylogenetic tree and Wirt Franklin (Microbiology Division, National Center for Toxological Research) for technical assistance.

\section{ADDENDUM}

We have received information from the RDP that the sequence whose accession number is M58682 was deleted from the RDP database as of 1 August 1993 because it was determined that this sequence is not an $F$. prausnitzii sequence but is probably the sequence of a contaminant. However, the sequence is still in the GenBank database, and other researchers used it after $1993(8,9)$.

\section{REFERENCES}

1. Brosius, J., J. L. Palmer, J. P. Kennedy, and H. F. Noller. 1978. Complete nucleotide sequence of a $16 \mathrm{~S}$ ribosomal RNA gene from Escherichia coli. Proc. Natl. Acad. Sci. USA 75:4801-4805.

2. Collins, M. D., P. A. Lawson, A. Willems, J. J. Cordoba, J. FernandezGarayzabal, P. Garcia, J. Cai, H. Hippe, and J. A. E. Farrow. 1994. The phylogeny of the genus Clostridium: proposal of five new genera and eleven new species combinations. Int. J. Syst. Bacteriol. 44:812-826.

3. Collins, M. D., S. Wallbanks, D. J. Lane, J. Shah, R. Nietupski, J. Smida, M. Dorsch, and E. Stackebrandt. 1991. Phylogenetic analysis of the genus Listeria based on reverse transcriptase sequencing of $16 \mathrm{~S}$ rRNA. Int. J. Syst. Bacteriol. 41:240-246.

4. Lawson, P. A., S. E. Gharbia, H. N. Shah, D. R. Clark, and M. D. Collins. 1991. Intrageneric relationships of members of the genus Fusobacterium as determined by reverse transcriptase sequencing of small-subunit rRNA. Int J. Syst. Bacteriol. 41:347-354.

5. Maidak, B. L., N. Larson, M. J. McCaughey, R. Overbeek, G. J. Olsen, K. Fogel, J. Blandy, and C. R. Woese. 1994. The Ribosomal Database Project. Nucleic Acids Res. 22:3485-3487.

6. Moore, W. E. C., and L. V. Holdeman. 1974. Human fecal flora: the normal flora of 20 Japanese-Hawaiians. Appl. Microbiol. 27:961-979.

7. Moore, W. E. C., L. V. Holdeman, and R. W. Kelley. 1984. Genus II. Fusobacterium, p. 602-662. In N. R. Krieg and J. G. Holt (ed.), Bergey's manual of systematic bacteriology, vol. 1. Williams \& Wilkins, Baltimore.

8. Neefs, J.-M., Y. V. de Peer, P. D. Rijk, S. Chapelle, and R. D. Wachter. 1993. Compilation of small ribosomal subunit RNA structures. Nucleic Acids Res. 21:3025-3049.

9. Nicholson, L. A., C. J. Morrow, L. A. Corner, and A. L. M. Hodgson. 1994. Phylogenetic relationship of Fusobacterium necrophorum A, AB, and B biotypes based upon 16S rRNA gene sequence analysis. Int. J. Syst. Bacteriol. 44:315-319.

10. Wang, R.-F., W.-W. Cao, W. L. Campbell, L. Hairston, W. Franklin, and C. E. Cerniglia. 1994. The use of PCR to monitor the population abundance of six human intestinal bacterial species in an in vitro semicontinuous culture system. FEMS Microbiol. Lett. 124:229-238.

11. Wang, R.-F., W.-W. Cao, and C. E. Cerniglia. 1995. Phylogenetic analysis of polycyclic aromatic hydrocarbon degrading mycobacteria by 16 S rRNA sequencing. FEMS Microbiol. Lett. 130:75-80. 